\title{
ON THE COMMUTATOR SUBGROUP OF THE ORTHOGONAL GROUP OVER THE 2-ADIC NUMBERS
}

\section{BARTH POLLAK}

1. Introduction. Let $V$ be a vector space of dimension $n$ over some field $k$ of characteristic $\neq 2$ with an orthogonal geometry as in [1, Chapter III]. Let $O(V)$ be the orthogonal group of $V, O^{\prime}(V)$ the subgroup of elements of determinant 1 and spinor-norm 1 and $\Omega(V)$ the commutator subgroup of $O(V)$. It is well known that $O^{\prime}(V)$ $=\Omega(V)$ if (i) $n \leqq 3$ or (ii) $V$ is isotropic. If $n>3$ and $V$ is anisotropic, this is no longer in general true. Our interest focuses on the case where $k$ is a local field (i.e., a field complete with respect to a discrete non-archimedean valuation with finite residue class field $\bar{k}$ ). Then it is well known that $V$ is isotropic if $n \geqq 5$. Hence we are left with the consideration of $n=4$ and $V$ anisotropic. In [4] Kneser states that in this case $O^{\prime}(V) \neq \Omega(V)$ and indeed it is not hard to show that $\left(O^{\prime}(V): \Omega(V)\right)=2$ if the characteristic of $\bar{k}>2$. It is the purpose of this note to prove that $O^{\prime}(V)=\Omega(V)$ when $k$ is the field of 2 -adic numbers. ${ }^{1}$

2. Preliminaries. Let us denote the symmetry with respect to the hyperplane perpendicular to the nonisotropic vector $A$ by $\tau_{A}$. We have

Proposition 1. Let $V$ have dimension $n$ and suppose $\sigma=\tau_{A_{1}} \tau_{A_{2}} \ldots$ $\tau_{A_{n}} \in O(V)$. Define a new space $V_{\sigma}=\left\langle A_{1}^{\prime}\right\rangle \perp\left\langle A_{2}^{\prime}\right\rangle \perp \cdots \perp\left\langle A_{n}^{\prime}\right\rangle$ by setting $\left(A_{i}^{\prime}\right)^{2}=A_{i}^{2}$ for $i=1,2, \cdots, n$. Suppose $V$ and $V_{\sigma}$ are isometric. Then $\sigma \in \Omega(V) \Leftrightarrow-1_{V_{0}} \in \Omega\left(V_{\sigma}\right)$.

Proof. Let $\phi: V_{\sigma} \rightarrow V$ be an isometry. Then $\phi O\left(V_{\sigma}\right) \phi^{-1}=O(V)$. Set $B_{i}=\phi A_{i}^{\prime}$ for $i=1,2, \cdots, n$. Then $\phi\left(-1_{V \sigma}\right) \phi^{-1}=\phi\left(\tau_{A_{1}^{\prime}} \tau_{A_{2}^{\prime}} \cdots \tau_{A_{n}^{\prime}}\right) \phi^{-1}$

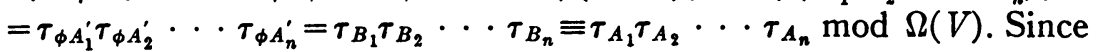
$\sigma=\tau_{A_{1}} \tau_{A_{2}} \cdots \tau_{A_{n}}$ we are through.

Proposition 2. Let $V$ be 4-dimensional and suppose $\sigma \in O^{\prime}(V)$. Then a necessary condition that $\sigma \notin \Omega(V)$ is that $\sigma$ have the form $\tau_{A_{1}} \tau_{A_{2}} \tau_{A_{3}} \tau_{A_{4}}$ and $A_{1}^{2}, A_{2}^{2}, A_{3}^{2}, A_{4}^{2}$ lie in distinct classes of $k^{*}$ modulo $k^{* 2}$.

Presented to the Society, January 25, 1962; received by the editors August 4, 1961.

${ }^{1} \mathrm{O}$. T. O'Meara informs me that he has proved this for any local field with characteristic $k=2$ using different methods. 
Proof. If $\sigma$ is the product of two symmetries, $\sigma=\tau_{A_{1}} \tau_{A_{2}}$, then $\sigma \in \Omega(V)$ as is shown in [1, Theorem 5.14]. Hence $\sigma$ has the form

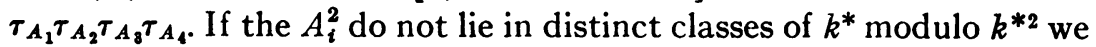
may assume (since the issue is $\bmod \Omega(V)$ ) that $A_{1}^{2}=A_{2}^{2}$. Then by Witt's Theorem, there exists $\lambda \in O(V)$ such that $A_{2}=\lambda A_{1}$. Thus $\sigma=\tau_{A_{1}} \tau_{A_{2}} \tau_{A_{3}} \tau_{A_{4}}=\tau_{A_{1}} \tau_{\lambda A_{1}} \tau_{A_{3}} \tau_{A_{4}}=\tau_{A_{1}} \lambda \tau_{A_{1}} \lambda^{-1} \tau_{A_{8}} \tau_{A_{4}} \equiv \tau_{A_{3}} \tau_{A_{4}} \bmod \Omega(V)$ and we are in the situation already dealt with at the beginning of the proof.

Although our main concern is over the field of 2 -adic numbers we now prove, for the sake of completeness, the following

THEOREM 1. Let $k$ be a local field with residue class field $\bar{k}$ of characteristic $>2$. Let $V$ be a 4-dimensional anisotropic space over $k$. Then $\left(O^{\prime}(V): \Omega(V)\right)=2$.

Proof. Since $\left(k^{*}: k^{* 2}\right)=4$, it follows immediately from Proposition 2 that $\left(O^{\prime}(V): \Omega(V)\right) \leqq 2$. We may choose as representatives for $k^{*}$ modulo $k^{* 2} 1, \nu, \pi, \nu \pi$ where $\nu$ is a nonsquare unit and $\pi$ is a prime. Since $V$ is anisotropic, we may write $V$ in the form $V=\left\langle A_{1}\right\rangle \perp\left\langle A_{2}\right\rangle$ $\perp\left\langle A_{3}\right\rangle \perp\left\langle A_{4}\right\rangle$ with $A_{1}^{2}=1, A_{2}^{2}=-\nu, A_{3}^{2}=\pi$ and $A_{4}^{2}=-\pi \nu$. Let $U=\left\langle A_{1}\right\rangle \perp\left\langle A_{2}\right\rangle$. There exists $B \in U$ such that $B^{2}=\nu$ as one easily verifies. Likewise, there exists $C \in U^{*}$ with $C^{2}=\pi \nu$. Set $\sigma=\tau_{A_{1}} \tau_{B} \tau_{A_{3}} \tau_{C}$. Then Dieudonne's technique (see [2, p. 93]) suitably modified shows that $\sigma \notin \Omega(V)$ and the theorem is proved.

3. Main result. We now assume that $V$ is a 4 -dimensional vector space over the field of 2-adic numbers. Furthermore we assume that $V$ possesses an orthogonal geometry that is anisotropic and note for future use that $V$ is unique up to isometry. (For a proof, see [3, Satz 7.3].)

$$
\text { Lemma. }-1_{V} \in \Omega(V) \text {. }
$$

Proof. We may set $V=\left\langle A_{1}\right\rangle \perp\left\langle A_{2}\right\rangle \perp\left\langle A_{3}\right\rangle \perp\left\langle A_{4}\right\rangle$ with $A_{i}^{2}=1$ for $i=1,2,3,4$. Then $-1_{V}=\tau_{A_{1}} \tau_{A_{2}} \tau_{A_{2}} \tau_{A_{4}}$ and hence is in $\Omega(V)$ by Proposition 2 .

TheOREM 2. $O^{\prime}(V)=\Omega(V)$.

Proof. It suffices to show that $O^{\prime}(V) \subseteq \Omega(V)$. Thus let $\sigma \in O^{\prime}(V)$. By Proposition 2 we may assume $\sigma=\tau_{A_{1}} \tau_{A_{2}} \tau_{A_{3}} \tau_{A_{4}}$ with the $A_{i}^{2}$ in different classes of $k^{*}$ modulo $k^{* 2}$. We may take $1,3,5,7,2,6,10,14$ as representatives of $k^{*}$ modulo $k^{* 2}$ and note that there are exactly 14 possibilities $\sigma_{i}$ for $\sigma$ defined by the set $\left\{A_{1}^{2}, A_{2}^{2}, A_{3}^{2}, A_{4}^{2}\right\}$. We list these and also note whether or not the corresponding $V_{\sigma_{i}}$ of Proposition 1 is anisotropic by an asterisk. 


\begin{tabular}{cl}
$i$ of $\sigma_{i}$ & $\left\{A_{1}^{2}, A_{2}^{2}, A_{3}^{2}, A_{4}^{2}\right\}$ \\
\hline 1 & $1,3,5,7$ \\
2 & $2,6,10,14$ \\
$3^{*}$ & $1,3,2,6$ \\
$4^{*}$ & $1,3,10,14$ \\
5 & $1,5,2,10$ \\
$6^{*}$ & $1,5,6,14$ \\
7 & $1,7,2,14$ \\
8 & $1,7,6,10$ \\
9 & $3,5,6,10$ \\
10 & $3,5,2,14$ \\
11 & $3,7,6,14$ \\
$12^{*}$ & $3,7,2,10$ \\
$13^{*}$ & $5,7,10,14$ \\
$14^{*}$ & $5,7,2,6$
\end{tabular}

By Proposition 1, the lemma and the fact, already noted, that all 4-dimensional anisotropic spaces are isometric we see that $\sigma_{3}, \sigma_{4}, \sigma_{6}$, $\sigma_{12}, \sigma_{13}$ and $\sigma_{14} \in \Omega(V)$. To complete the proof first note that $\sigma \equiv \tau \bmod \Omega(V) \Leftrightarrow \sigma \tau \in \Omega(V)$. Now $\sigma_{1} \sigma_{3} \equiv \sigma_{14} \bmod \Omega(V)$. But $\sigma_{3}$ and $\sigma_{14}$ are in $\Omega(V)$. Hence, $\sigma_{1} \in \Omega(V)$. Similar computations show that the remaining $\sigma_{i}$ lie in $\Omega(V)$ and the theorem is proved.

\section{REFERENCES}

1. E. Artin, Geometric algebra, Interscience, New York, 1957.

2. J. Dieudonne, On the orthogonal groups over the rational field, Ann. of Math. (2) 54 (1951), 85-93.

3. M. Eichler, Quadratische Formen und orthogonale Gruppen, Springer, Berlin, 1952.

4. M. Kneser, Orthogonale Gruppen über algebraischen Zahlkörpern, J. Reine Angew. Math. 196 (1956), 213-220.

\section{SyRACUSE UNIVERSITY AND}

INSTITUTE FOR DEFENSE ANALYSES 